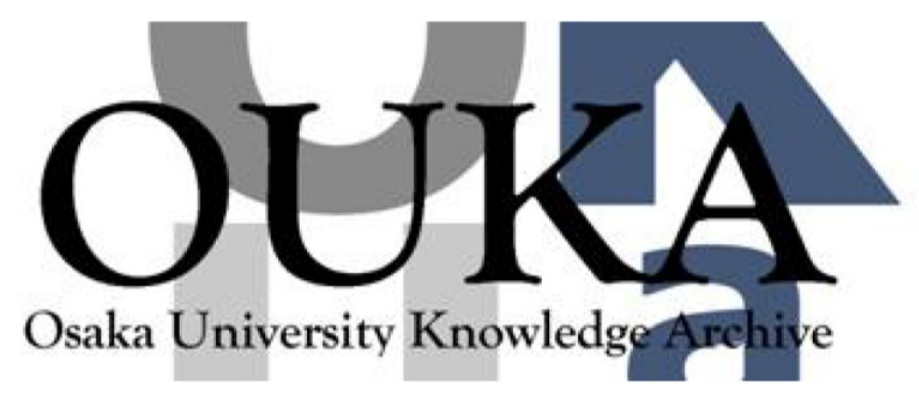

\begin{tabular}{|c|l|}
\hline Title & $\begin{array}{l}\text { Spin-Current-Driven Spin Pumping in Rashba } \\
\text { Spin-Orbit Coupled Systems : A Spin Torque } \\
\text { Effect }\end{array}$ \\
\hline Author(s) & Hattori, Kiminori \\
\hline Citation & $\begin{array}{l}\text { Journal of the Physical Society of Japan. 78(8) } \\
\text { p. } 084703\end{array}$ \\
\hline Issue Date & $2009-08$ \\
\hline oaire:version & AM \\
\hline URL & https://hdl.handle. net/11094/2951 \\
\hline rights & \\
\hline Note & \\
\hline
\end{tabular}

Osaka University Knowledge Archive : OUKA

https://ir. Library. osaka-u. ac. jp/

Osaka University 


\title{
Spin-Current-Driven Spin Pumping in Rashba Spin-Orbit Coupled Systems: A Spin Torque Effect
}

\author{
Kiminori HATTORI* \\ Department of Systems Innovation, Graduate School of Engineering Science, \\ Osaka University, Toyonaka, Osaka 560-8531, Japan
}

There exists a direct connection between spin torque and spin current in two-dimensional electron systems with linear in momentum Rashba spin-orbit (SO) coupling. In terms of the spin-current continuity equation, we show that the spin torque of this type generates a divergent spin current due to spin injection, which we call the spin-current-driven spin pumping. We quantitatively investigate the spin pumping from SO coupled systems in contact with spin-polarized reservoirs using the nonequilibrium Green’s function formalism, demonstrating that the spin torque effect efficiently produces a pure spin current which is orders of magnitude larger than the spin Hall current.

KEYWORDS: spin-orbit coupling, spin torque, spin pumping, spin transport, two-dimensional system

*E-mail: hattori@ee.es.osaka-u.ac.jp 


\section{Introduction}

Spintronics is a burgeoning field in condensed matter physics which aims to harness electron spin in addition to charge in solid-sate systems. In this field, an active interest exists in a generation of a pure spin current without any net charge flow in nonmagnetic metals or semiconductors. Many ideas for achieving this goal have been proposed to date. Several approaches are based on purely magnetic means, including spin pumping from paramagnetic quantum dots ${ }^{1-3)}$ or finite-sized conductors in paramagnetic resonance, ${ }^{4)}$ as well as a spin battery consisting of a ferromagnet with precessing magnetization attached to nonmagnetic metals or semiconductors. ${ }^{5)}$ A route to manipulate spin current by electrical means is provided by the spin-orbit (SO) coupling in conventional semiconductors and quantum heterostructures. Most intensively investigated is the spin Hall effect arising in SO coupled systems, ${ }^{6,7)}$ which constitutes a spin analog to the conventional charge Hall effect, and generates a transverse spin flux in response to a longitudinal electric field or an unpolarized charge current. Parametric quantum pumping by cyclic variations of system parameters has attracted a renewed interest for spin current generation in the presence of SO coupling. ${ }^{8-10)}$ Spin pumping mechanisms exploiting the linear in momentum Rashba SO coupling in two-dimensional electron systems lacking the structural inversion symmetry have also been devised, which operate with a dynamic modulation of the SO coupling strength due to an oscillating gate potential, ${ }^{11,12)}$ as well as the electric dipole spin resonance under an oscillating in-plane electric field. ${ }^{13)}$

Generally, electron spin is not a conserved quantity so that the spin-current continuity equation may involve the spin torque contribution as a source term. A physically transparent consequence of spin nonconservation is a possibility of spin pumping when the driving spin 
torque is activated. In this paper, we investigate the spin torque effect in Rashba SO coupled systems in contact with spin-polarized reservoirs. The spin torque in the Rashba system is exerted by spin injection, giving rise to a spin-current-driven spin pumping. The spin current generation due to spin injection is not new but has been found in a previous theoretical study, ${ }^{14)}$ showing that in a four-terminal bridge geometry a longitudinal spin current driven by a virtual spin bias induces a transverse spin current with perpendicular polarization. In the literature, however, its physical origin is not addressed. In this paper, we uncover the underlying spin torque physics, and propose a spin battery operating with an ordinary electric bias.

\section{Theoretical Analysis}

Throughout this paper we shall work in units where $\hbar=e=1$. We consider the Rashba SO coupled system in the $x y$ plane described by the Hamiltonian $H=\mathbf{p}^{2} / 2 m+U(\mathbf{r})+\omega_{\mathrm{so}} \cdot \mathbf{S}$, where $\mathbf{p}$ is the canonical momentum operator, $\mathbf{S}$ is the spin operator, $\boldsymbol{\omega}_{\mathrm{SO}}=\lambda \mathbf{p} \times \mathbf{e}_{z}$ is the momentum-dependent spin precession frequency, $m$ is the electron mass, $\lambda$ is the SO coupling strength, and $\mathbf{e}_{\mu}(\mu=x, y, z)$ is the unit vector in Cartesian coordinates. The electrostatic potential $U$, which can deal with lateral confinement or nonmagnetic scatterers, is incorporated for generality. In terms of the Heisenberg equation, we derive the kinetic velocity operator $\mathbf{v}=\dot{\mathbf{H}}=\mathbf{v}_{0}+\mathbf{v}_{\mathrm{SO}}$, which consists of the canonical velocity $\mathbf{v}_{0}=\mathbf{p} / m$ and the spin-dependent anomalous velocity $\mathbf{v}_{\mathrm{SO}}=\lambda \mathbf{e}_{z} \times \mathbf{S}$ due to SO coupling. The same procedure defines the spin

torque operator $\dot{S}=\omega_{\mathrm{so}} \times \mathrm{S}$. The spin torque of this type stems from the spin nonconservation due to SO coupling, and is distinct in nature from the field-induced torque ${ }^{3,4)}$ or the spin-transfer torque. ${ }^{15-17)}$ In this study, we specifically address the spin torque physics and its 
related effects in the SO coupled system.

The Schrödinger equation $i \partial_{t} \psi=H \psi$ in terms of the fermion field operator $\psi$ leads to the continuity equation for the spin density operator $\rho^{\mu}=\psi^{\dagger} S^{\mu} \psi$ and the spin current density operator $\mathbf{j}^{\mu}=\operatorname{Re} \psi^{\dagger} S^{\mu} \mathbf{v} \psi$, expressed as

$$
\frac{\partial}{\partial} \rho^{\mu}(\mathbf{r}, t)+\nabla \cdot \mathbf{j}^{\mu}(\mathbf{r}, t)=g^{\mu}(\mathbf{r}, t)
$$

where $\operatorname{Re} A=\left(A+A^{\dagger}\right) / 2$, and the operator $g^{\mu}=\operatorname{Re} \psi^{\dagger} S^{\prime \mu} \psi$ represents the spin torque density. The spin current density $\mathbf{j}^{\mu}$ is decomposed into $\mathbf{j}_{0, \mathrm{SO}}^{\mu}=\operatorname{Re} \psi^{\dagger} S^{\mu} \mathbf{v}_{0, \mathrm{SO}} \psi$. Note that $\mathbf{j}_{\text {SO }}^{z}=0$, and hence $\mathbf{j}^{z}=\mathbf{j}_{0}^{z}$. In terms of the kinetic contributions $\mathbf{j}_{0}^{\mu}$, each component of spin torque density can be explicitly written as

$$
\begin{aligned}
& g^{x}(\mathbf{r}, t)=-j_{0, x}^{z}(\mathbf{r}, t) / L_{\mathrm{SO}}, \\
& g^{y}(\mathbf{r}, t)=-j_{0, y}^{z}(\mathbf{r}, t) / L_{\mathrm{SO}}, \\
& g^{z}(\mathbf{r}, t)=\left[j_{0, x}^{x}(\mathbf{r}, t)+j_{0, y}^{y}(\mathbf{r}, t)\right] / L_{\mathrm{SO}},
\end{aligned}
$$

where $L_{\mathrm{SO}}=1 / m \lambda$ is the spin precession length (over which spin precesses by one radian). The direct connection between spin torque and spin current is a unique property inherent to the linear in momentum SO coupling. It should be noticed that eqs. (1) and (2) are independent of $U$ and hold for any potential profiles.

The spin-current continuity equation for the SO coupled system contains an important physical implication for the spin torque effect. In the steady-state, eq. (1) reduces to $\nabla \cdot \mathbf{j}^{\mu}=g^{\mu}$. The local continuity provides a conservation law for spin flux 


$$
\oint_{C} \mathbf{j}^{\mu}(\mathbf{r}) \cdot \mathbf{n} d r=\int_{A} g^{\mu}(\mathbf{r}) d^{2} r
$$

where the closed loop $C$ encloses the area $A$, and $\mathbf{n}$ is the outward-directed unit vector normal to $C$. Thus, the total outflow of spin current is equal to the integrated spin torque. In terms of eq. (2), the spin torque is generated in the presence of spin currents, giving rise to a spin-current-driven spin pumping. The spin pumping process involves a polarization conversion, i.e., the out-of-plane (in-plane) spin torque is produced by the in-plane (out-of-plane) polarized spin current.

For definiteness, we specifically consider a four-terminal bridge geometry as illustrated in Fig. 1. The model consists of a central rectangular sample with SO coupling and four leads without SO coupling attached on all four sides of the sample. The two longitudinal leads (labeled by 1 and 3) are $S^{x}$-polarized whereas the two transverse leads (labeled by 2 and 4 ) are unpolarized. This configuration corresponds to a Datta-Das spin transistor ${ }^{18)}$ to which additional unpolarized transverse leads are attached. The charge current with $S^{x}$-polarization flows between the two longitudinal leads when subjected to a certain voltage bias. The spin torque $g^{z}$ exerted by the spin injection generates a divergent spin current with $S^{z}$-polarization in the sample region, which flows into the leads connected to the sample. If the electrochemical potentials in the transverse leads are suitably adjusted to prevent charge currents, pure spin currents are extracted. Thus, the spin torque effect enables a stationary spin-battery operation, which is distinguished from conventional spin-pumping mechanisms that exploit time-dependent external fields. ${ }^{1-5,8-13)}$ Note that the spin-current-driven spin pumping occurs effectively in a finite-sized sample smaller than the spin precession length $L_{\mathrm{so}}$. It is trivially 
expected for an infinitely-large sample that the spin torque substantially averages out because of a continuous spin precession.

In the tight-binding representation on a square lattice with lattice spacing $a$, the Hamiltonian describing the sample region is expressed as

$$
H=-t_{0} \sum_{\mathbf{r}, \sigma} \sum_{ \pm} c_{\mathbf{r} \sigma}^{\dagger}\left(c_{\mathbf{r} \pm \mathbf{a} \sigma}+c_{\mathbf{r} \pm \mathbf{b} \sigma}\right)+\sum_{\mathbf{r}, \sigma} \varepsilon_{\mathbf{r}} c_{\mathbf{r} \sigma}^{\dagger} c_{\mathbf{r} \sigma}-2 i t_{\mathrm{SO}} \sum_{\mathbf{r}, \sigma, \sigma^{\prime} \pm} \sum_{\mathbf{r} \sigma} c_{\mathbf{r}}^{\dagger}\left( \pm S_{\sigma \sigma^{\prime}}^{x} c_{\mathbf{r} \pm \mathbf{b} \sigma^{\prime}} \mp S_{\sigma \sigma^{\prime}}^{y} C_{\mathbf{r} \pm \mathbf{a} \sigma^{\prime}}\right)
$$

where $c_{\mathbf{r} \sigma}$ is the fermionic annihilation operator of an electron at position $\mathbf{r}$ with spin $\sigma$, $\mathbf{a}=a \mathbf{e}_{x}$ and $\mathbf{b}=a \mathbf{e}_{y}$ are the unit lattice vectors, $t_{0}=1 / 2 m a^{2}$ is the hopping energy, $\varepsilon_{\mathbf{r}}=U(\mathbf{r})+4 t_{0}$ is the effective on-site energy, and $t_{\mathrm{sO}}=\lambda / 4 a$ is the SO coupling energy. In the lattice model, the spin density $\rho^{\mu}$ and the spin current density $\mathbf{j}^{\mu}=\mathbf{j}_{0}^{\mu}+\mathbf{j}_{\text {so }}^{\mu}$ are formulated as

$$
\begin{aligned}
& \rho^{\mu}(\mathbf{r})=\frac{1}{a^{2}} \sum_{\sigma, \sigma^{\prime}} c_{\mathbf{r} \sigma}^{\dagger} S_{\sigma \sigma^{\prime}}^{\mu} c_{\mathbf{r} \sigma^{\prime}}, \\
& \mathbf{j}_{0}^{\mu}(\mathbf{r})=\frac{t_{0}}{a} \operatorname{Im} \sum_{\sigma, \sigma^{\prime} \pm} c_{\mathbf{r} \sigma^{\prime}} S_{\sigma \sigma^{\prime}}^{\mu}\left( \pm c_{\mathbf{r} \pm \mathbf{a} \sigma^{\prime}} \mathbf{e}_{x} \pm c_{\mathbf{r} \pm \mathbf{b} \sigma^{\prime}} \mathbf{e}_{y}\right), \\
& \mathbf{j}_{\mathrm{SO}}^{x}(\mathbf{r})=\frac{t_{\mathrm{SO}}}{2 a} \operatorname{Re} \sum_{\sigma} \sum_{ \pm} c_{\mathbf{r} \sigma}^{\dagger} c_{\mathbf{r} \pm \mathbf{b} \sigma} \mathbf{e}_{y}, \\
& \mathbf{j}_{\mathrm{SO}}^{y}(\mathbf{r})=-\frac{t_{\mathrm{SO}}}{2 a} \operatorname{Re} \sum_{\sigma} \sum_{ \pm} c_{\mathbf{r} \sigma}^{\dagger} c_{\mathbf{r} \pm \mathbf{a} \sigma} \mathbf{e}_{x},
\end{aligned}
$$

where $\operatorname{Im} A=\left(A-A^{\dagger}\right) / 2 i$. In eq. (5), $\mathbf{j}^{\mu}(\mathbf{r})$ describes the local spin current on site $\mathbf{r}$, which is equivalent to the average of bond spin currents $\mathbf{j}^{\mu}\left(\mathbf{r}, \mathbf{r}^{\prime}\right)$ from the site $\mathbf{r}$ to its nearest neighbor sites $\mathbf{r}^{\prime}{ }^{19)}$ The lattice version of spin torque density $g^{\mu}(\mathbf{r})$ is simply given by eq. (2) with adopting the lattice version of $\mathbf{j}_{0}^{\mu}(\mathbf{r})$. It is shown from the Heisenberg equation 
$\partial_{t} \rho^{\mu}=-i\left[\rho^{\mu}, H\right]$ that these lattice expressions satisfy the continuity equation, eq. (1).

It is convenient for the numerical analysis to introduce the nonequilibrium Green's function formalism, ${ }^{20-22)}$ which employs the retarded and lesser Green's functions defined by

$$
\begin{aligned}
& G_{\mathbf{r} \sigma, \mathbf{r}^{\prime} \sigma^{\prime}}^{+}\left(t, t^{\prime}\right)=-i\left\langle\left\{c_{\mathbf{r} \sigma}(t), c_{\mathbf{r}^{\prime} \sigma^{\prime}}^{\dagger}\left(t^{\prime}\right)\right\}\right\rangle \theta\left(t-t^{\prime}\right), \\
& G_{\mathbf{r} \sigma, \mathbf{r}^{\prime} \sigma^{\prime}}^{<}\left(t, t^{\prime}\right)=i\left\langle c_{\mathbf{r}^{\prime} \sigma^{\prime}}^{\dagger}\left(t^{\prime}\right) c_{\mathbf{r} \sigma}(t)\right\rangle,
\end{aligned}
$$

respectively. In stationary situations, these double-time correlation functions depend only on the time difference $t-t^{\prime}$. The retarded Green's function is Fourier transformed into

$$
G^{+}(\varepsilon)=\left[\varepsilon-H-\Sigma^{+}(\varepsilon)\right]^{-1}
$$

with $\Sigma^{+}=\Sigma_{\alpha} \Sigma_{\alpha}^{+}$, where $\Sigma_{\alpha}^{+}=V_{\alpha} g_{\alpha}^{+} V_{\alpha}^{\dagger}$ is the retarded self-energy due to lead $\alpha, V_{\alpha}$ is the hopping matrix connecting lead $\alpha$ with the sample, and $g_{\alpha}^{+}$is the retarded function of the isolated lead. The lesser Green’s function satisfies the Keldysh equation,

$$
G^{<}(\varepsilon)=G^{+}(\varepsilon) \Sigma^{<}(\varepsilon) G^{-}(\varepsilon)
$$

with $G^{-}=\left(G^{+}\right)^{\dagger}$ and $\Sigma^{<}=\Sigma_{\alpha} \Sigma_{\alpha}^{<}$, where $\Sigma_{\alpha}^{<}=i f_{\alpha} \Gamma_{\alpha}$ is the lesser self-energy due to lead $\alpha, f_{\alpha}$ is the Fermi function in lead $\alpha$, and $\Gamma_{\alpha}=-2 \operatorname{Im} \Sigma_{\alpha}^{+}$. The local physical quantities such as the spin current density $\left\langle\mathbf{j}^{\mu}(\mathbf{r})\right\rangle$ and the spin torque density $\left\langle g^{\mu}(\mathbf{r})\right\rangle$ are calculated directly from the equal-time correlation function $G^{<}(t, t)=(2 \pi)^{-1} \int_{-\infty}^{\infty} d \varepsilon G^{<}(\varepsilon)$. This expression can be cast into a linear form for small deviations from equilibrium at zero temperature. ${ }^{23)}$ The spin- $S^{\mu}$ 
component of spin current flowing through lead $\alpha$ into the sample region is defined by $J_{\alpha}^{\mu}=-\left\langle\mathcal{S}_{\alpha}^{\dot{\mu}}\right\rangle$, where the operator $S_{\alpha}^{\mu}=\sum_{\mathbf{r} \in \alpha, \sigma, \sigma^{\prime}} C_{\mathbf{r} \sigma}^{\dagger} S_{\sigma \sigma^{\prime}}^{\mu} c_{\mathbf{r} \sigma^{\prime}}$ describes the total spin in lead $\alpha$. Assuming spin conservation in lead $\alpha$ and correspondingly $\left[S^{\mu}, \Sigma_{\alpha}^{+}\right]=0$, the Heisenberg equation for $S_{\alpha}^{\mu}$ leads to a linearized Landauer-Büttiker formula for terminal spin current,

$$
J_{\alpha}^{\mu}=\frac{1}{2 \pi} \sum_{\beta} T_{\alpha \beta}^{\mu}\left(\mu_{\alpha}-\mu_{\beta}\right)
$$

with $T_{\alpha \beta}^{\mu}=\operatorname{Tr}\left(S^{\mu} \Gamma_{\alpha} G^{+} \Gamma_{\beta} G^{-}\right)$, where $\mu_{\alpha}$ denotes the electrochemical potential in lead $\alpha{ }^{13)}$ Equation (10) is analogous to the well-known formula for terminal charge current, $J_{\alpha}=(2 \pi)^{-1} \sum_{\beta} T_{\alpha \beta}\left(\mu_{\alpha}-\mu_{\beta}\right)$ with $T_{\alpha \beta}=\operatorname{Tr}\left(\Gamma_{\alpha} G^{+} \Gamma_{\beta} G^{-}\right)$, which is derived from the Heisenberg equation for the number operator $N_{\alpha}=\sum_{\mathrm{r} \in \alpha, \sigma} c_{\mathrm{r} \sigma}^{\dagger} c_{\mathrm{r} \sigma}$ on the basis of charge conservation. ${ }^{20,22)}$

The spin polarization of lead may be formally represented either by the spectral function $\rho_{\alpha}\left(=-2 \operatorname{Im} g_{\alpha}^{+}\right)$or by the coupling matrix $V_{\alpha}$. In the former picture, we use $\rho_{\alpha}=\sum_{\sigma} \eta_{\sigma}(p) P_{\sigma}^{v} \rho_{\alpha}^{(0)} \quad$ with $\quad \eta_{\sigma}(p)=(1+\sigma p) /(1+|p|) \quad$ for $\quad S^{v}$-polarization. Here, $\rho_{\alpha}^{(0)}$ corresponds to the spectral function of unpolarized lead, $P_{\sigma}^{v}=1 / 2+\sigma S^{v}$ is the projection matrix onto the spin- $S^{v}$ eigenvector, and $p(-1 \leq p \leq 1)$ stands for the degree of spin polarization. For example, $\rho_{\alpha}=\rho_{\alpha}^{(0)}$ for $p=0$ while $\rho_{\alpha}=P_{\uparrow, \downarrow}^{v} \rho_{\alpha}^{(0)}$ for $p= \pm 1$. The former picture is equivalent to the latter one where the coupling matrix is defined by $V_{\alpha}=\sum_{\sigma} \sqrt{\eta_{\sigma}(p)} P_{\sigma}^{\nu} V_{\alpha}^{(0)}$, insofar as transport properties are concerned (i.e., both pictures lead to the same self-energy $\Sigma_{\alpha}^{+}$). It should be noticed that eq. (10) for spin- $S^{\mu}$ current is valid when the probe lead $\alpha$ is unpolarized $(p=0)$ or $S^{\mu}$-polarized $(p \neq 0$ and $\mu=v)$. The present treatment mimics a spin-asymmetry in the density of states around the Fermi level in the Stoner model of ferromagnetism. This phenomenological approach is useful for analyzing the spin 
torque effect, which is fundamentally characterized by the sample size, the spin precession length, as well as the injected spin current, as shown in the following.

\section{Numerical Calculation}

In the rest of this paper, we explain the results of numerical calculations performed for a quantitative study of the spin torque effect. Hereafter, the bracket \langle\rangle representing the nonequilibrium statistical average is omitted for simplicity. As shown in Fig. 1, the model system is basically comprised of a central square sample of size $L \times L$ with SO coupling and four semi-infinite leads of the same width $L$ without SO coupling. The spin polarization of two longitudinal leads $\alpha=1,3$ is varied in the range $0 \leq p \leq 1$ while two transverse leads $\alpha=2,4$ remain unpolarized. In this model, we examine the terminal spin current $J_{\alpha}^{\mu}$ in comparison with the total spin torque $G^{\mu}=a^{2} \sum_{\mathrm{r}} g^{\mu}(\mathbf{r})$. Throughout the calculation, the condition $J_{2,4}=0$ is imposed on terminal charge currents so that pure spin currents flow in transverse leads. To avoid tediousness, in what follows we explain the longitudinal and transverse spin currents in terms of the conserved components: $J_{\ell-}^{\mu}=\left(J_{1}^{\mu}-J_{3}^{\mu}\right) / 2$ and $J_{t-}^{\mu}=\left(J_{2}^{\mu}-J_{4}^{\mu}\right) / 2$, and the nonconserved ones: $J_{\ell+}^{\mu}=-\left(J_{1}^{\mu}+J_{3}^{\mu}\right)$ and $J_{t+}^{\mu}=-\left(J_{2}^{\mu}+J_{4}^{\mu}\right)$. Similar definitions also apply to terminal charge currents, which have only the conserved component $J_{\ell-}=\left(J_{1}-J_{3}\right) / 2$. All the numerical results are normalized by a factor $\left(\mu_{1}-\mu_{3}\right) / 2 \pi$. Accordingly, the spin current and the spin torque shown below have a dimension of spin conductance (in units of $e / 2 \pi$ ). In addition to the standard model, we consider a modified structure where a normal region with $\lambda=p=0$ is inserted between each lead and the sample for investigating the microscopic details of spin current flow. In the normal region, the local 
spin current is conserved and nondivergent, i.e., $\nabla \cdot \mathbf{j}^{\mu}=0$, in contrast to the general relation $\nabla \cdot \mathbf{j}^{\mu}=g^{\mu}$ involving the local spin torque in the sample region. In the following, the hopping energy is taken as the energy unit ( $\left.t_{0}=1\right)$, and the lattice spacing as the length unit ( $\left.a=1\right)$. The SO coupling energy is normally set to be $t_{\mathrm{so}}=0.01$, which corresponds to the spin precession length $L_{\mathrm{SO}}=50$. The sample size $L$ is chosen to be smaller than $L_{\mathrm{SO}}$ so that the spin precession does not significantly affect the spin torque effect observed in the present calculation.

Before discussing the numerical results, it may be appropriate to summarize the general relations for terminal spin currents. In the present model, the electrostatic potential has $C_{2}$ symmetry around the $x, y, z$-axis, i.e., $U(x, y)=U(x,-y)=U(-x, y)=U(-x,-y)$ in the absence of random impurities. Consequently, the total Hamiltonian for $p=0$ is invariant under each of the following unitary transformations: ${ }^{14)}$ (i) $x \rightarrow-x, z \rightarrow-z, S^{y} \rightarrow-S^{y}, S^{z} \rightarrow-S^{z}$, $y \rightarrow-y, z \rightarrow-z, S^{x} \rightarrow-S^{x}, S^{z} \rightarrow-S^{z}$, and (iii) $x \rightarrow-x, y \rightarrow-y, S^{x} \rightarrow-S^{x}, S^{y} \rightarrow-S^{y}$, leading to the symmetry relation $J_{\ell \pm}^{x}=J_{\ell-}^{y}=J_{\ell \pm}^{z}=J_{t \pm}^{x}=J_{t-}^{y}=J_{t+}^{z}=0$ via the Landauer-Büttiker formalism. On the other hand, the symmetries under transformations (ii) and (iii) are broken for $p \neq 0$. In this case, the relevant relation is only $J_{\ell+}^{x}=J_{t \pm}^{x}=0$. These analytical results help to interpret the numerical results for clean ballistic systems described below.

For $p=0$, the spin Hall effect arises in response to the unpolarized charge current. What is shown below is that the resulting spin-Hall current brings about a spin torque effect. Figure 2 (a) illustrates the conserved transverse spin current $J_{t-}^{z}$ calculated as a function of the Fermi energy $\varepsilon_{F}$. A small oscillation is due to the subband formation in the finite-sized system subjected to lateral confinement. The remaining nonzero spin-currents $J_{\ell+}^{y}$ and $J_{t+}^{y}$ are displayed in Fig. 2 (b), along with the total spin torque $G^{y}$. These nonconserved spin currents 
appear with opposite signs, implying that the spin- $S^{y}$ current flows from transverse leads to longitudinal leads, and vice versa (depending on $\varepsilon_{F}$ ). However, it is notable that the local spin current $\mathbf{j}^{y}$ is not conserved in the sample region where the finite spin torque $g^{y}$ is generated by the local spin-Hall current $j_{y}^{z}=j_{0, y}^{z}$ [see, eq. 2(b)]. As explicitly stated in eq. (3), the total outflow of spin currents is identical to the total spin torque. Hence, the terminal spin currents should obey the relation $J_{\ell+}^{y}+J_{t+}^{y}=G^{y}$. The expected relation holds among the associated numerical results exactly. Figure 3 shows the spatial profiles of local spin current and local spin torque calculated for $\varepsilon_{F}=0.1$, demonstrating that the spin current $\mathbf{j}^{\mathrm{z}}$ flowing toward $-y$ generates the spin torque $g^{y}$ in the sample region, and simultaneously the spin current $\mathbf{j}^{y}$ is drawn out of transverse leads and flows into longitudinal leads.

The terminal spin currents vary as $J_{\ell+}^{y}, J_{t+}^{y} \propto \lambda$ and $J_{t-}^{z} \propto \lambda^{2}$ with the SO coupling strength $\lambda$ when $L<L_{\mathrm{SO}}$ (not shown). The quadratic dependence of $J_{t-}^{z}$ is naturally expected from its driving spin-force, ${ }^{24,25)}$ which is also quadratic in $\lambda$. It appears that the spin currents $J_{\ell+}^{y}$ and $J_{t+}^{y}$ linear in $\lambda$ are of different origin. The occurrence of these spin currents may be ascribed to a nonuniform spin accumulation $\rho^{y}$ in the SO coupled region, as suggested previously. ${ }^{26)}$

For $p \neq 0$, the spin-current-driven spin pumping is expected to occur. The spin-charge ratio of conserved longitudinal currents $P=J_{\ell-}^{x} / J_{\ell-}$ increases monotonically with $p$, and reaches the maximum $P=1 / 2$ at $p=1$ (corresponding to half-metallic leads). In the presence of the conserved longitudinal spin current $J_{\ell-}^{x}$, the nonconserved transverse spin current $J_{t+}^{z}$ emerges. As shown in Fig. 4 (a), $J_{t+}^{z}$ grows continuously with $p$. In addition to the pumped spin current $J_{t+}^{z}$, the nonzero spin currents $J_{t-}^{z}$ and $J_{t \pm}^{y}$ are detected in unpolarized transverse leads. The spin Hall current $J_{t-}^{z}$ and the spin polarization current $J_{t+}^{y}$ occurring due to the longitudinal charge current $J_{\ell-}$ are on the same order of magnitude for $p=0$. As compared to 
these ordinary spin currents, the pumped spin current $J_{t+}^{z}$ observed for $p=1$ is orders of magnitude larger (compare Figs. 2 and 4). The conserved transverse spin current $J_{t-}^{y}$ (which does not exist in the case of $p=0$ ) behaves as $J_{t-}^{y} \cong \theta_{\mathrm{SO}} J_{t+}^{\mathrm{z}} / 8$ (not shown), and is closely related to the pumped spin current $J_{t+}^{z}$, where $\theta_{\mathrm{SO}}=L / L_{\mathrm{SO}}$ is the spin precession angle for travel distance $L$. This relation suggests that $J_{t-}^{y}$ originates from the spin precession in the $y z$ plane accompanying $J_{t+}^{z}$. Figure 4 (b) explains that the pumped spin current $J_{t+}^{z}$ probed in the transverse leads is appreciably lower than the total spin torque $G^{z}$, indicating that the spin- $S^{z}$ current flows partly into longitudinal leads, in view of eq. (3). This interpretation is supported by the spatial distributions of local spin current and local spin torque shown in Fig. 5. The spin current $\mathbf{j}^{x}$ flows toward $x$ and generates the spin torque $g^{z}$ in the sample region. The spin current $\mathbf{j}^{2}$ flows divergently, and is finally absorbed into all four leads. The total outflow of spin- $S^{z}$ currents is directly calculated from the line integral of $\mathbf{j}^{z}$ along a closed loop across the normal regions. Comparing it to the total spin torque $G^{z}$, the conservation law for spin pumping, eq. (3), is validated exactly.

The total spin torque generated in the sample region, which corresponds to the maximum output spin current, can be roughly estimated from the input spin current. To simplify the matter, we here assume a uniform flow of spin currents such that $\mathbf{j}^{\mu}=\left(J_{x}^{\mu} \mathbf{e}_{x}+J_{y}^{\mu} \mathbf{e}_{y}\right) / L$ for a sufficiently weak SO coupling. Each component of total spin torque $G^{\mu}$ is then written as $G^{x}=-\theta_{\mathrm{SO}} J_{x}^{z}, G^{y}=-\theta_{\mathrm{SO}} J_{y}^{z}$, and $G^{z}=\theta_{\mathrm{SO}}\left(J_{x}^{x}+J_{y}^{y}\right)$. For instance, the spin torque induced by the spin Hall current is described by $G^{y}=\theta_{\mathrm{SO}} J_{t-}^{z}$. A similar treatment applies to the spin torque due to spin injection, giving $G^{z}=\theta_{\mathrm{SO}} J_{\ell-}^{x}$ for the particular configuration illustrated in Fig. 1 . These relations are merely an approximation assuming uniform spin currents but satisfactorily account for the numerical results, providing a simple way to evaluate the spin torque from the 
input spin current.

In terms of the spin-torque mechanism we address, the four-terminal geometry analogous to a spin-Hall bridge is not a prerequisite for the spin-current-driven spin pumping. To demonstrate this, additional numerical results for a three-terminal configuration are shown in Figs. 6 and 7. The three-terminal model assumed in the calculation corresponds to the model illustrated in Fig. 1 with simply removing the bottom lead 4, which preserves the symmetry under transformation (i) so that $J_{\ell+}^{X}=J_{2}^{x}=0$ (in this case, $J_{t \pm}^{\mu}$ is not proper for describing transverse spin currents). Figure 6 compares the pumped spin currents $-J_{2}^{z}$ observed in the three- and four-terminal models, showing that the output spin currents in both cases are similar in magnitude. The microscopic details of spin-current flow and spin-torque distribution are explained in Fig. 7. Physical insights into the spin torque effect are the same as those found in the four-terminal model, i.e., the spin torque $g^{z}$ exerted by the spin current $\mathbf{j}^{x}$ creates a divergent flow of the spin current $\mathbf{j}^{z}$. The conservation law for spin pumping is numerically verified by calculating the line integral of $\mathbf{j}^{z}$ and the total spin torque $G^{z}$. The spin torque effect occurring efficiently in different structures exemplifies its extended applicability to spin pumping.

Finally, we investigate the disorder effect due to nonmagnetic impurities. In this study, the static disorder is taken into account by a random on-site potential $U$ uniformly distributed in the range $-W / 2 \leq U \leq W / 2$ (for which $C_{2}$ symmetry is broken). Figure 8 shows the spin Hall current $J_{t-}^{z}$ for $p=0$ and the pumped spin current $J_{t+}^{z}$ for $p=1$ calculated as a function of the disorder strength $W$, in comparison with the charge currents $J_{\ell-}$ computed simultaneously. In this figure, the numerical results are normalized by the values in the clean limit $(W=0)$. It is easily found that although both spin currents tend to be suppressed for a strong disorder, there is 
a significant difference between them. The spin Hall current decays more rapidly than the charge current and almost disappears at $W \cong 2$. This feature is implied from a vanishing spin-Hall conductivity for an arbitrary weak disorder in the thermodynamical limit, ${ }^{27,28)}$ and also from an extended Drude model including the spin force. ${ }^{29)}$ The Drude model predicts $\mathbf{j}^{z}=\left(m \lambda^{2} \tau / 4\right) \mathbf{j} \times \mathbf{e}_{z}$ to second order in $\lambda$ for Rashba systems, where $\tau$ represents the momentum relaxation time. This suggests that $\mathbf{j}^{2}$ decreases faster than $\mathbf{j}$ for a shorter $\tau$. In contrast, the pumped spin current slowly decreases and remains finite even at $W \cong 2$. Actually, the pumped spin current and the charge current exhibit a similar fall-off property. This observation is indicative that the spin torque effect is controlled mainly by the injected spin current even for a strong disorder.

\section{Conclusions}

The spin torque effect is investigated in multi-terminal systems with the Rashba SO coupling in the center region. The spin torque exerted by spin injection generates a divergent spin current in the SO coupled region which flows into the attached leads, enabling the spin-current-driven spin pumping in stationary situations. In terms of the spin-current continuity equation, the conservation law for spin pumping is immediately derived, which states that the total outflow of pumped spin current is identical to the total spin torque. The

spin-current-driven spin pumping effectively occurs for finite-sized systems with the spin precession angle $\theta_{\mathrm{SO}}=L / L_{\mathrm{SO}}$ smaller than unity, and produces much larger spin current compared to the spin Hall effect.

It is straightforward to extend the present theory to other linear in momentum SO 
couplings. For instance, in the case of the Dresselhaus SO coupling described by $H_{\mathrm{D}}=\beta\left(p_{x} S^{x}-p_{y} S^{y}\right)$, the local spin torque is expressed as $g^{x}=-j_{0, y}^{z} / L_{\mathrm{D}}, g^{y}=-j_{0, x}^{\mathrm{z}} / L_{\mathrm{D}}$, and $g^{z}=\left(j_{0, y}^{x}+j_{0, x}^{y}\right) / L_{\mathrm{D}}$, where $L_{\mathrm{D}}=1 / m \beta$. The physical consequences drawn from these expressions (which become equivalent to eq. (2) when exchanging flow directions $x \leftrightarrow y$ ) are very similar to those derived for the Rashba SO coupling. Moreover, it is notable that metallic surfaces may exhibit the Rashba SO coupling due to the loss of inversion symmetry. ${ }^{30-32)}$ The spin injection from ferromagnetic metals into the Rashba SO coupled surface states constitutes a promising spin-pumping scheme, in addition to the spin injection from ferromagnetic semiconductors or metals into the semiconductor heterostructures with the Rashba or Dresselhaus SO coupling. We expect the theoretical results obtained in this study to be useful for exploiting the spin torque effect in spintronics.

\section{Acknowledgement}

This work was supported by Grant-in-Aid for Scientific Research (No. 21540320) from the Japan Society for the Promotion of Science. 


\section{REFERENCES}

1) P. Zhang, Q.-K. Xue, and X. C. Xie: Phys. Rev. Lett. 91 (2003) 196602.

2) B. Wang, J. Wang, and H. Guo: Phys. Rev. B 67 (2003) 092408.

3) K. Hattori: Phys. Rev. B 78 (2008) 155321.

4) K. Hattori: Phys. Rev. B 75 (2007) 205302.

5) A. Brataas, Y. Tserkovnyak, G. E. W. Bauer, and B. I. Halperin: Phys. Rev. B 66 (2002) 060404(R).

6) S. Murakami, N. Nagaosa, and S.-C. Zhang: Phys. Rev. B 69 (2004) 235206.

7) J. Sinova, D. Culcer, Q. Niu, N. A. Sinitsyn, T. Jungwirth, and A. H. MacDonald: Phys. Rev. Lett. 92 (2004) 126603.

8) P. Sharma and P. W. Brouwer: Phys. Rev. Lett. 91 (2003) 166801.

9) M. Governale, F. Taddei, and R. Fazio: Phys. Rev. B 68 (2003) 155324.

10) C. Li, Y. Yu, Y. Wei, and J. Wang: Phys. Rev. B 75 (2007) 035312.

11) A. G. Mal'shukov, C. S. Tang, C. S. Chu, and K. A. Chao: Phys. Rev. B 68 (2003) 233307.

12) L. Y. Wang, C. S. Tang, and C. S. Chu: Phys. Rev. B 73 (2006) 085304.

13) K. Hattori: J. Phys. Soc. Jpn. 77 (2008) 034707.

14) Y. Xing, Q.-F. Sun, and J. Wang: Phys. Rev. B 75 (2007) 075324.

15) J. C. Slonczewski: J. Magn. Magn. Mater. 159 (1996) L1.

16) Z. Li and S. Zhang: Phys. Rev. Lett. 92 (2004) 207203.

17) Y. Tserkovnyak, A. Brataas, and G. E. W. Bauer: J. Magn. Magn. Mater. 320 (2008) 1282.

18) S. Datta and B. Das: Appl. Phys. Lett. 56 (1990) 665.

19) B. K. Nikolic, L. P. Zarbo, and S. Souma: Phys. Rev. B 73 (2006) 075303. 
20) S. Datta: Electronic Transport in Mesoscopic Systems (Cambridge University Press, Cambridge, 1995).

21) G. D. Mahan: Many-Particle Physics (Kluwer Academic, Dordrecht/Plenum, New York, 2000).

22) H. Haug and A.-P. Jauho: Quantum Kinetics in Transport and Optics of Semiconductors (Springer-Verlag, New York, 2007).

23) K. Hattori and H. Okamoto: Phys. Rev. B 74 (2006) 155321.

24) S.-Q. Shen: Phys. Rev. Lett. 95 (2005) 187203.

25) B. Zhou, L. Ren, and S.-Q. Shen: Phys. Rev. B 73 (2006) 165303.

26) B. K. Nikolic, L. P. Zarbo, and S. Souma: Phys. Rev. B 72 (2005) 075361.

27) J. Inoue, G. E. W. Bauer, and L. W. Molenkamp: Phys. Rev. B 70 (2004) 041303(R).

28) S. S. Mandal and A. Sensharma: Phys. Rev. B 78 (2008) 205313.

29) E. M. Chudnovsky: Phys. Rev. Lett. 99 (2007) 206601.

30) S. LaShell, B. A. McDougall, and E. Jensen: Phys. Rev. Lett. 77 (1996) 3419.

31) O. Krupin, G. Bihlmayer, K. Starke, S. Gorovikov, J. E. Prieto, K. Dobrich, S. Blugel, and G. Kaindl: Phys. Rev. B 71 (2005) 201403(R).

32) C. R. Ast, J. Henk, A. Ernst, L. Moreschini, M. C. Falub, D. Pacile, P. Bruno, K. Kern, and M. Grioni: Phys. Rev. Lett. 98 (2007) 186807. 


\section{FIGURE CAPTIONS}

FIG. 1

(Color online) Schematic diagram for a four-terminal system with Rashba SO coupling in the center rectangular region. The two longitudinal leads 1 and 3 are $S^{x}$-polarized while the two transverse leads 2 and 4 are unpolarized. The spin-polarized charge current flowing between longitudinal leads generates the spin torque in the SO coupled region. The spin torque induces a divergent spin- $S^{z}$ current, which can be detected in transverse leads.

FIG. 2

(Color online) Terminal spin currents and total spin torque versus Fermi energy for $p=0$. Two panels show (a) $J_{t-}^{z}$, (b) $J_{\ell+}^{y}, J_{t+}^{y}$, and $G^{y}$. The parameters used in the calculation are indicated in the figure.

FIG. 3

(Color online) Local spin currents and local spin torque for $p=0$. Three panels show (a) $\mathbf{j}^{z}$, (b)

$g^{y}$, and (c) $\mathbf{j}^{y}$. The parameters used in the calculation are $t_{\mathrm{so}}=0.01$ and $\varepsilon_{F}=0.1$. The four rectangular arms attached to the central square sample are the normal regions where $\lambda=p=0$.

FIG 4

(Color online) Terminal spin currents and total spin torque versus Fermi energy for $0 \leq p \leq 1$. Two panels show (a) $J_{t+}^{z}$ for $p$ varying in 0.2 steps, and (b) $J_{\ell-}^{x}, J_{t+}^{z}$, and $G^{z}$ for $p=1$. The parameters used in the calculation are indicated in the figure. 


\section{FIG. 5}

(Color online) Local spin currents and local spin torque for $p=1$. Three panels show (a) $\mathbf{j}^{x}$, (b) $g^{z}$, and (c) $\mathbf{j}^{z}$. The parameters used in the calculation are $t_{\mathrm{SO}}=0.01$ and $\varepsilon_{F}=0.1$. The four rectangular arms attached to the central square sample are the normal regions where $\lambda=p=0$.

FIG. 6

(Color online) Pumped spin currents $-J_{2}^{z}$ in three- and four-terminal systems as a function of Fermi energy. The parameters used in the calculation are indicated in the figure. Insets illustrate the models assumed in the calculation.

\section{FIG. 7}

(Color online) Local spin currents and local spin torque in three-terminal system with $p=1$. Three panels show (a) $\mathbf{j}^{x}$, (b) $g^{z}$, and (c) $\mathbf{j}^{z}$. The parameters used in the calculation are $t_{\mathrm{SO}}=0.01$ and $\varepsilon_{F}=0.1$. The three rectangular arms attached to the central square sample are the normal regions where $\lambda=p=0$.

\section{FIG. 8}

(Color online) Normalized terminal currents as a function of disorder strength. Two panels show (a) $J_{t-}^{z}$ and $J_{\ell-}$ for $p=0$, and (b) $J_{t+}^{z}$ and $J_{\ell-}$ for $p=1$. The parameters used in the calculation are indicated in the figure. In the calculation, the disorder average is performed over 1000 random configurations. 


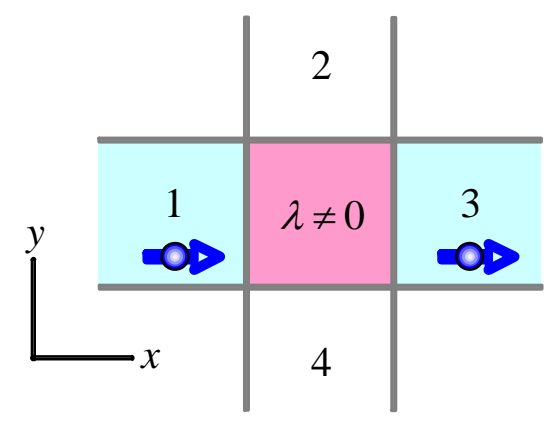

FIG. 1 


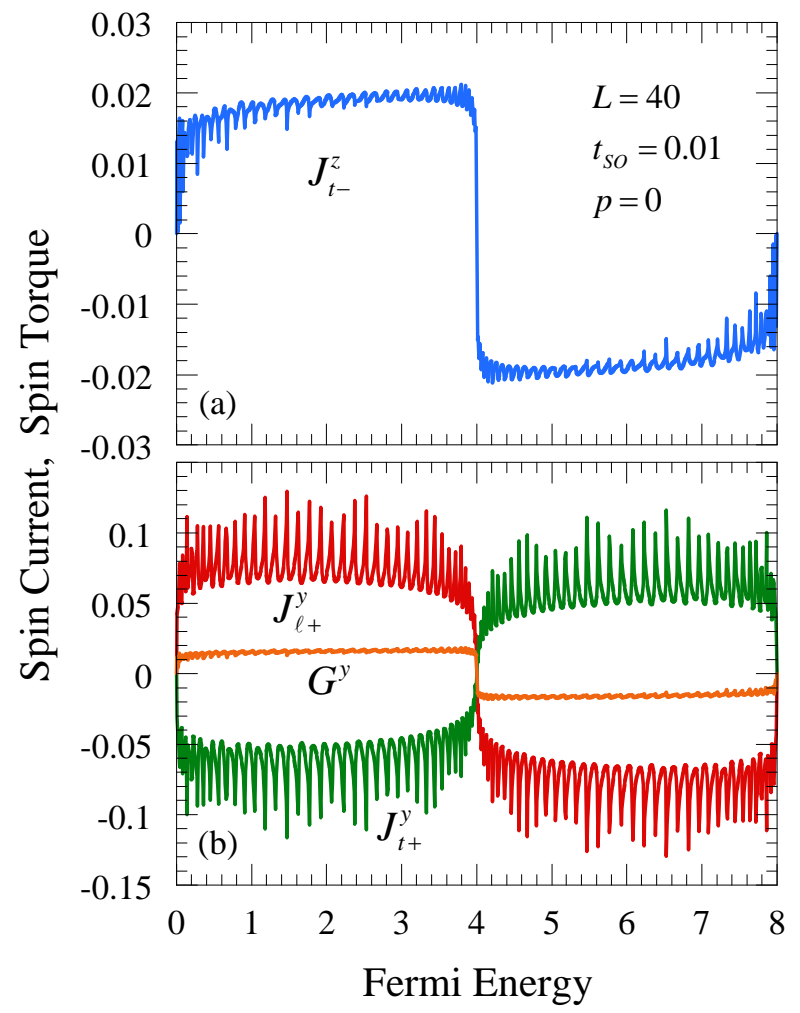

FIG. 2 

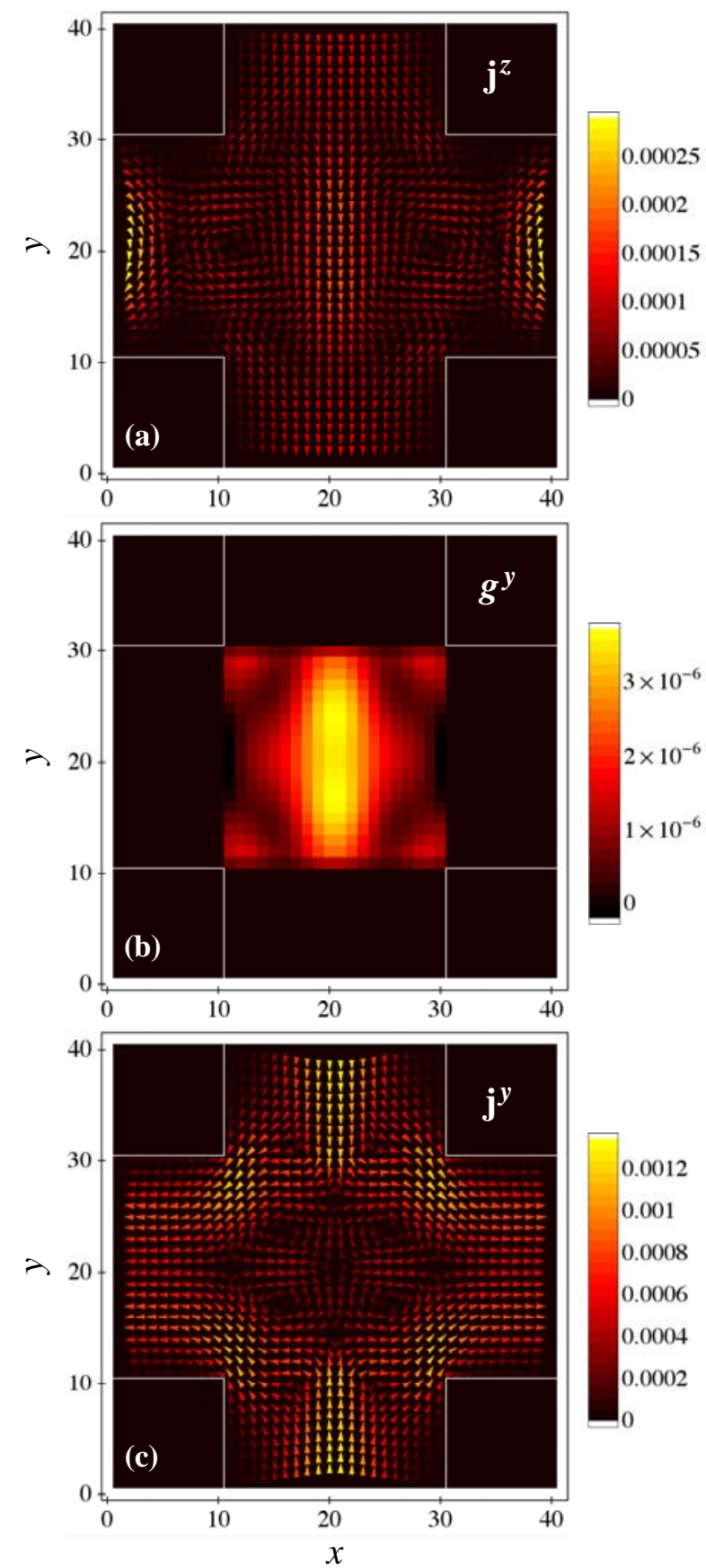

FIG. 3 


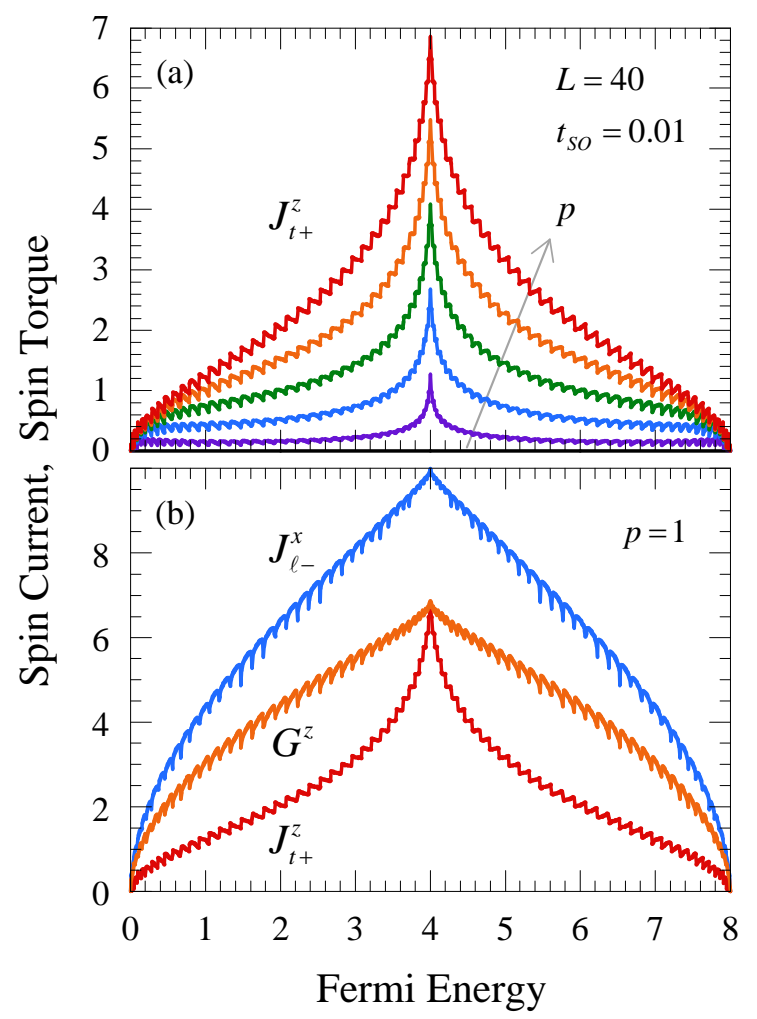

FIG. 4 


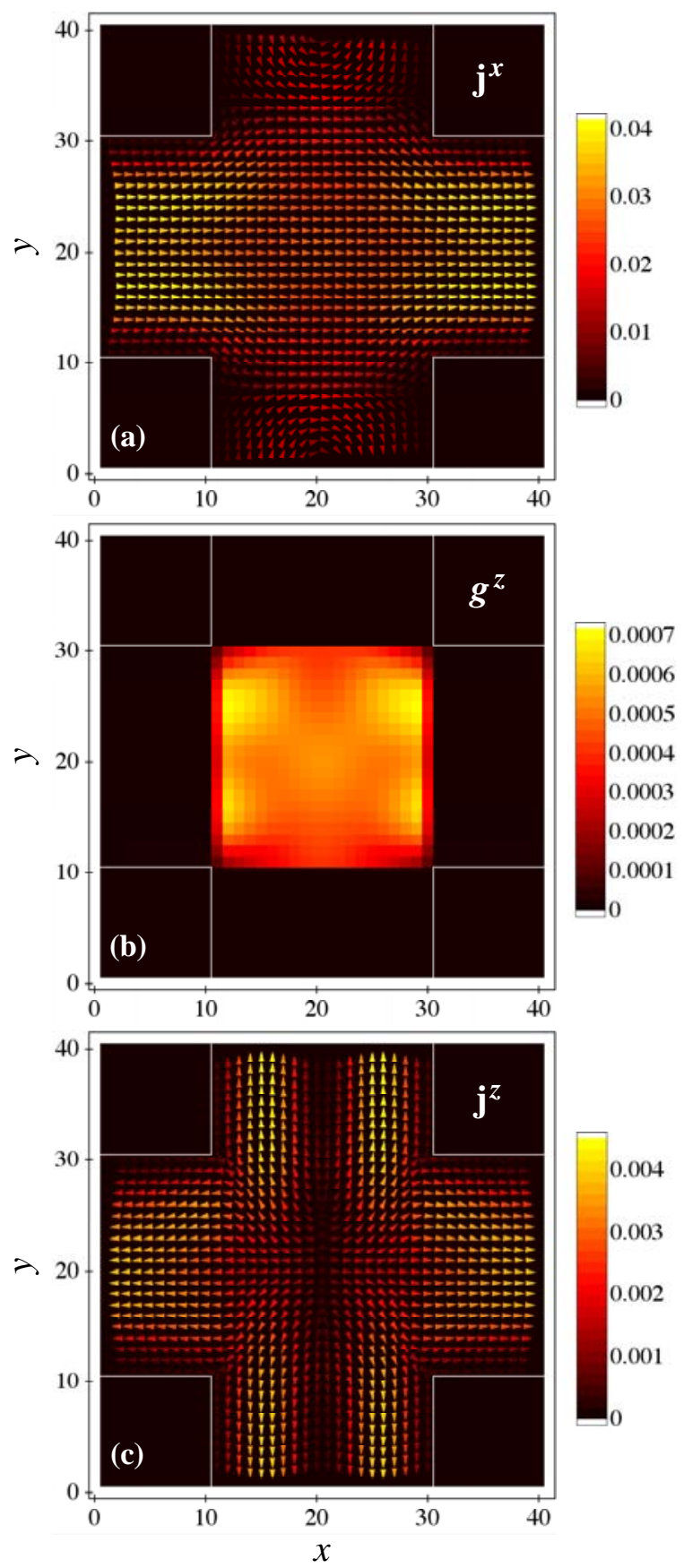

FIG. 5 


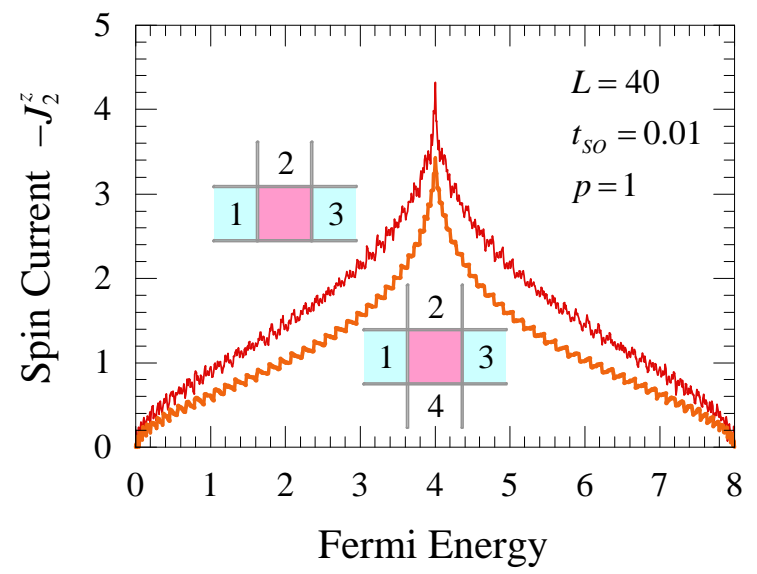

FIG. 6 

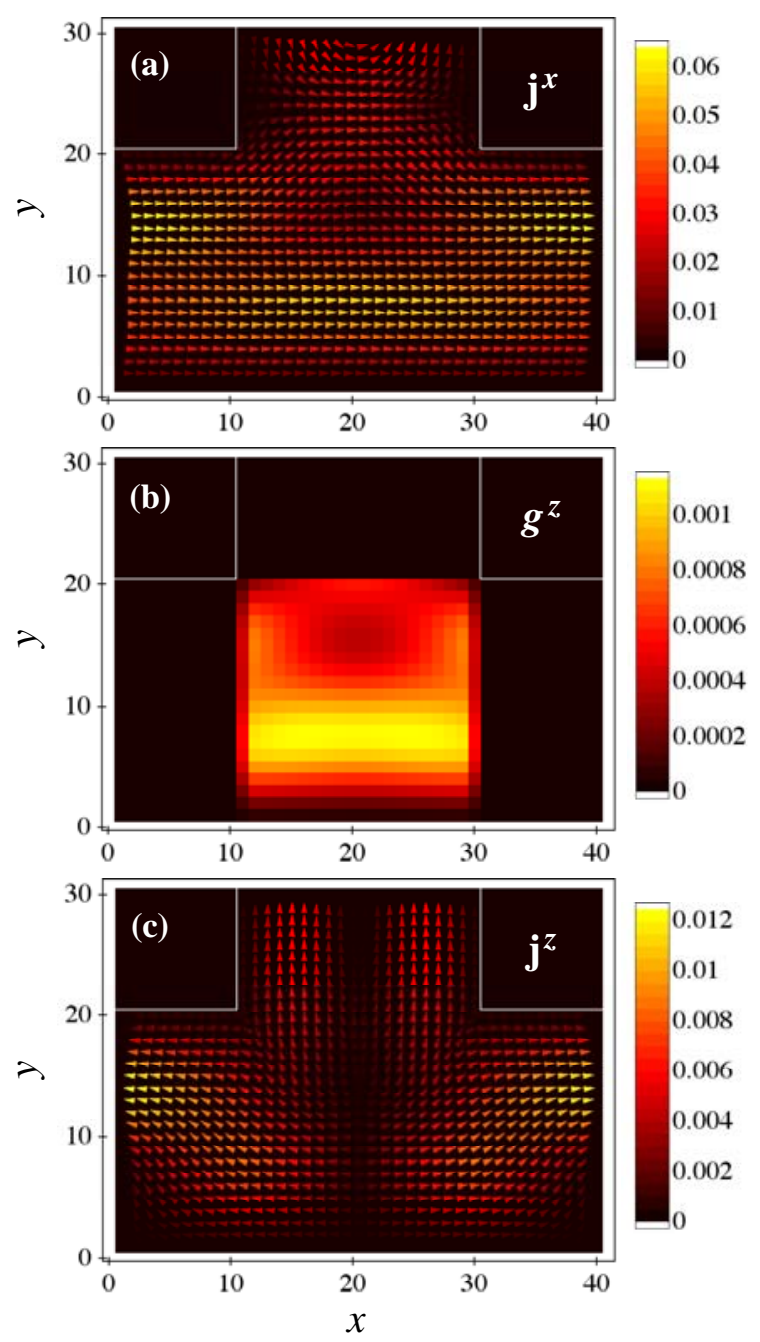

FIG. 7 


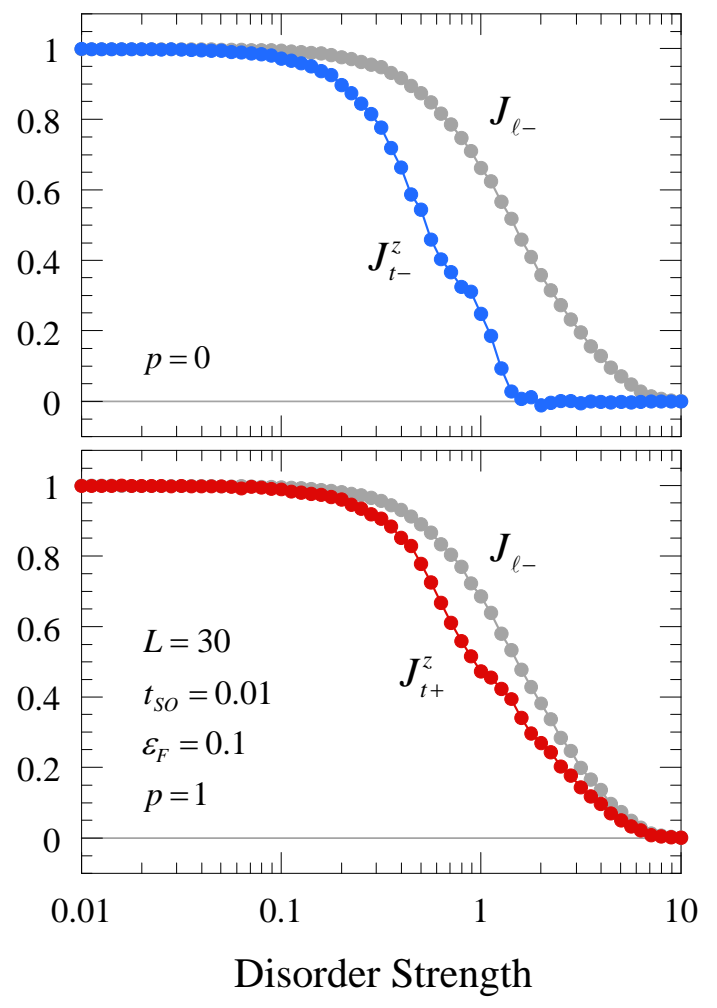

FIG. 8 